\title{
Strategy for alignment of electron beam trajectory in LEReC cooling section
}

\author{
S. Seletskiy, M. Blaskiewicz, A. Fedotov, \\ D. Kayran, J. Kewisch, R. Michnoff, I. Pinayev
}

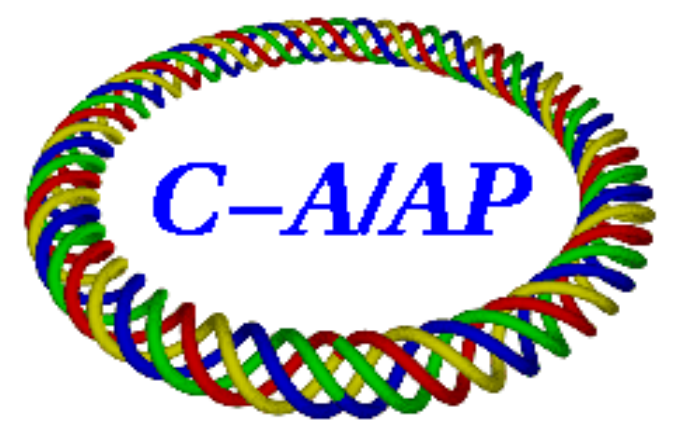

\section{Collider-Accelerator Department Brookhaven National Laboratory \\ Upton, NY 11973}

\section{U.S. Department of Energy}

\author{
Office of Science, Office of Nuclear Physics
}

Notice: This document has been authorized by employees of Brookhaven Science Associates, LLC under Contract No. DE-SC0012704 with the U.S. Department of Energy. The United States Government retains a nonexclusive, paid-up, irrevocable, world-wide license to publish or reproduce the published form of this document, or allow others to do so, for United States Government purposes. 


\title{
Strategy for alignment of electron beam trajectory in LEReC cooling section
}

\author{
S. Seletskiy, M. Blaskiewicz, A. Fedotov, D. Kayran, J. Kewisch, R. Michnoff, \\ I. Pinayev \\ 9/23/2016
}

\section{Introduction}

LEReC cooling section (CS) consists of two $20 \mathrm{~m}$ long parts (Fig. 1) each containing 6 solenoids with weak trajectory correctors placed inside the solenoids and the BPMs located downstream of each solenoid. The distance between solenoid centers is $3 \mathrm{~m}$. The solenoids are used to minimize the scalloping of the electron beam envelope.

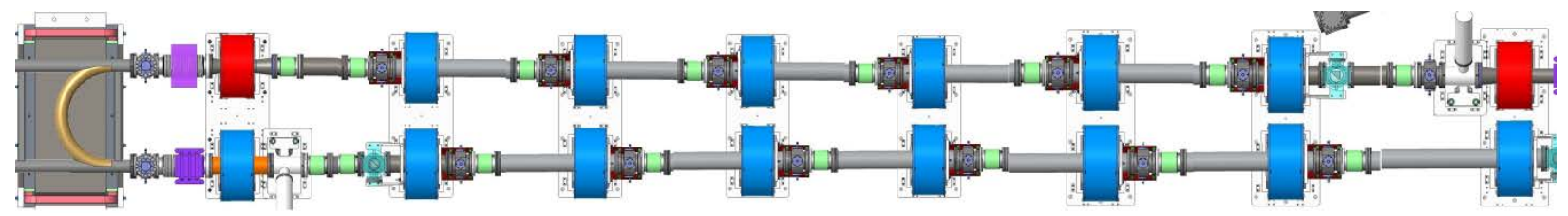

Figure 1: Schematic of LEReC cooling section.

We envision the following basic steps for the LEReC CS commissioning and demonstration of the cooling:

0. Prerequisites: electron beam energy and energy spread are optimized, cooling section is properly shielded.

1. Aligning CS BPMs with respect to the ion beam.

2. Aligning the trajectory of low charge electron beam by zeroing the e-beam displacement in each BPM. For properly shielded cooling section this step automatically guarantees [1] that electron beam trajectory angles throughout most of the CS are below $100 \mathrm{urad}$.

3. Performing beam-based alignment of the CS solenoids.

4. Setting proper focusing for high-charge e-beam in the CS.

5. Combining high-charge e-beam with low charge ion bunches in the cooling section. The charge of ions shall be low enough to have a negligible effect on e-beam focusing. We expect to observe cooling of the low charge ion beam at this step.

6. Increase ion charge and adjust the CS solenoids focusing respectively to obtain ions cooling with design parameters.

To obtain the cooling it is required to keep the overall RMS electron angles in the cooling section below 100 urad. Possible mechanical misalignment, such as shift and inclination (see Fig. 2), of the CS solenoids can cause an unacceptable misalignment of the e-beam trajectory 
with respect to the ideal trajectory set by ions. Therefore, it is critical to go through step 3 in the procedure outlined above.

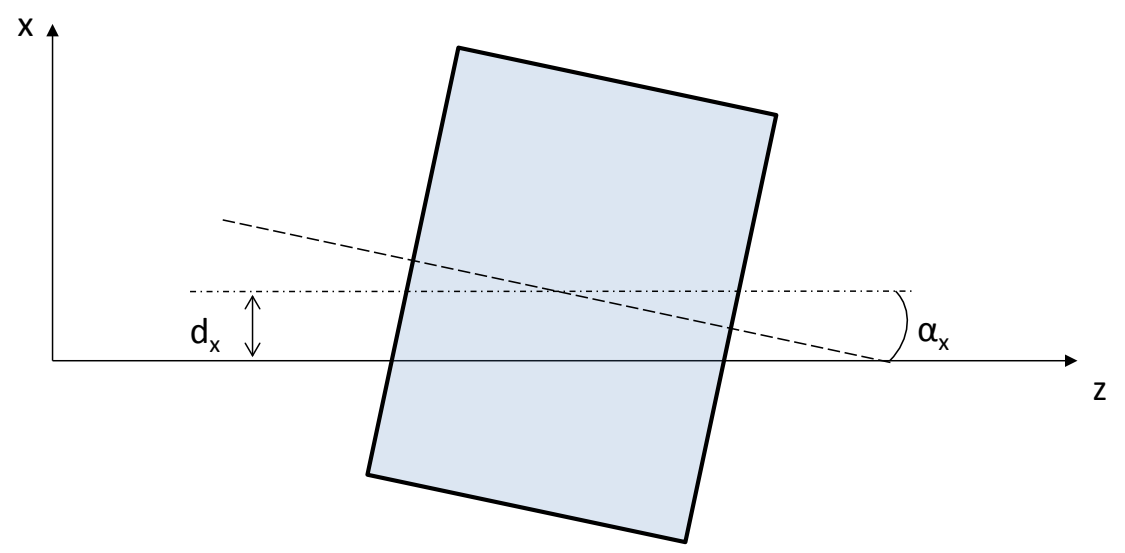

Figure 2: A solenoid magnetic center is shifted by $d_{x}$ from and the solenoid is inclined by an angle $\alpha_{x}$ with respect to the optimal electron trajectory.

In this paper we will briefly describe step 1 of the procedure. Step 2 is trivial (for the properly shielded CS) and is covered by [1]. Most of our discussions will be devoted to step 3 of the procedure. Steps 4 and especially 6 require a separate study and will be covered in a future publication.

\section{CS BPM alignment}

The electron beam consists of macro-bunches repeated with $9 \mathrm{MHz}$ frequency, which coincides with the frequency of stored ion bunches traveling through the cooling section. Each macro-bunch of electron consists of 30 bunches repeated with the frequency of $704 \mathrm{MHz}$.

We suggest the following general algorithm for the alignment of the CS BPMs:

1. Send the e-beam with desired current through the CS and observe the intensity of the CS BPM signals with $9 \mathrm{MHz}$ band pass filter.

2. Stop the electron beam and inject the ion beam of such current that the CS BPM signals have the same intensity that they did for the e-beam.

3. Set the desired ion trajectory through the cooling section using well-calibrated existing RHIC BPMs and centering the ion beam in the nearby RHIC quads.

4. Measure the positions of the ions in the CS BPMs with $9 \mathrm{MHz}$ band pass filter. We will call these positions BPM9io.

5. "Turn off" ions, "turn on" electrons and measure electron beam positions in the CS BPMs using $9 \mathrm{MHz}$ filter. We call these positions BPM $\mathrm{Be}_{9 \mathrm{e}}$.

6. Switch to $704 \mathrm{MHz}$ filter, which will be routinely used to measure positions of electrons, and measure electron positions $\mathrm{BPM}_{704 \mathrm{eO}}$. 
Apparently, the ion and electron beam trajectories coincide when $\mathrm{BPM}_{9 \mathrm{e} 0}=\mathrm{BPM}_{9 \mathrm{i} 0}$. Then, the optimal (absolute zero) electron beam position measured with $704 \mathrm{MHz}$ filter is:

$$
\mathrm{BPM}_{704 \mathrm{e}}=\mathrm{BPM}_{704 \mathrm{e} 0}+\left(\mathrm{BPM}_{9 \mathrm{i} 0}-\mathrm{BPM}_{9 \mathrm{e} 0}\right)
$$

\section{Electron beam motion in solenoid field}

Each CS solenoidal module [2] consists of the main solenoid and two weak correcting antisolenoids (bucking coils), which reduce the longitudinal "tails" of the field. The field profile of solenoids was modelled [3] and measured for each module [4]. The measured field profiles repeat the profile predicted by the model. Figure 3 show the modelled field profile for maximum solenoid current. In our simulations we use this field profile.

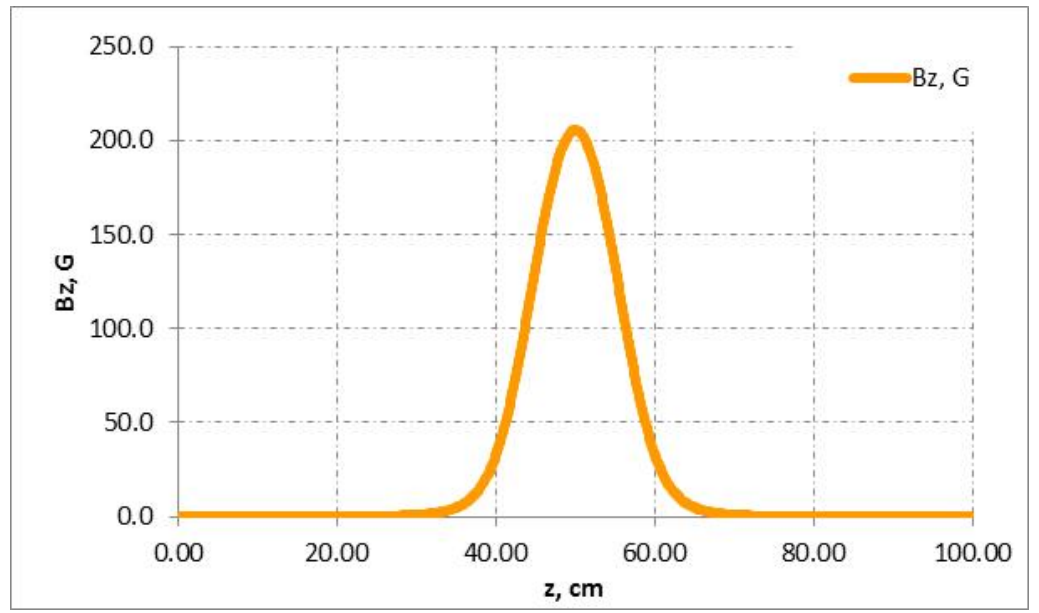

Figure 3: Simulated field of the CS solenoidal module.

To simulate motion of the e-beam centroid in magnetic field we use an approach suggested in [5]. Introducing $\xi=x+i y, \theta=\theta_{x}+i \theta_{y}$ and $B_{\perp}=B_{x}+i B_{y}$ we can write equation of motion in a paraxial approximation as:

$$
\left\{\begin{array}{c}
\xi^{\prime}=\theta \\
\theta^{\prime}=\frac{i}{B \rho}\left(B_{\perp}-B_{z} \theta\right)
\end{array}\right.
$$

Here a prime defines differentiation with respect to longitudinal coordinate $z$.

In absence of additional transverse fields $\left(B_{\perp}\right)$ the off axis field is due to the non-uniformity of $B_{z}$ only and from Maxwell equations: 


$$
B_{\perp}=-\frac{\xi}{2} \frac{d B_{z}}{d z}
$$

For the stable numerical solution Eq. (1) must be solved with an implicit method, which gives:

$$
\left\{\begin{array}{c}
\xi^{n+1}=\xi^{n}+\frac{\Delta z}{2}\left(\theta^{n}+\theta^{n+1}\right) \\
\theta^{n+1}=\frac{\theta^{n}+i \frac{\Delta z}{2 B \rho}\left(B_{\perp}{ }^{n}+B_{\perp}{ }^{n+1}-B_{z}{ }^{n} \theta^{n}\right)}{1+i \frac{\Delta z}{2 B \rho} B_{z}{ }^{n+1}}
\end{array}\right.
$$

In this paper we use (3) to simulate beam motion through the solenoid module. For the transverse fields at each step of simulation we always use a sum of trajectory correctors' fields and the fields defined by (2).

\section{Requirements to beam based alignment of CS solenoids}

As it was mentioned in Section 1 the overall RMS angles of the electrons shall be below 100 urad.

In this paper we will consider the case of $1.6 \mathrm{MeV}$ electron beam. For such beam, for its nominal bunch charge of $100 \mathrm{pC}$, the CS solenoid strength of $\sim 70 \mathrm{G}$ is required. We might require electron bunches of higher charge and therefore require the stronger solenoids. Apparently, since the highest peak field of solenoids is $\sim 200 \mathrm{G}$, the limit on acceptable mechanical displacements of the CS solenoid calculated for such field will be a reasonable overestimate (with some safety factor) of the tolerance of solenoid alignment.

Simulating beam motion through the field shown in Fig. 3 according to Eq. 3 we find that the electron beam trajectory will acquire 100 urad angle from solenoid inclination of $0.5 \mathrm{mrad}$ or from solenoid displacement of $0.5 \mathrm{~mm}$.

Therefore, the CS solenoid inclination with respect to the design beam trajectory must be measured and corrected with accuracy much better than 0.5 mrad. The displacement of the CS solenoid magnetic center with respect to the design beam trajectory must be measured and corrected with accuracy much better than $0.5 \mathrm{~mm}$.

\section{Hard-edge approximation}

The proper hard-edge approximation of the measured real field shall provide both the proper beam focusing and $x-y$ rotation. That is, the hard-edge solenoid field $(B)$ and length $(L)$ shall satisfy the following relations: 


$$
\begin{gathered}
B=\frac{\int B(z)^{2} d z}{\int B(z) d z} \\
L=\frac{\left(\int B(z) d z\right)^{2}}{\int B(z)^{2} d z}
\end{gathered}
$$

Using (4) we get $L=18.906 \mathrm{~cm}$ and $B=0.70743 \cdot \max (\mathrm{B}(\mathrm{z}))$. Figure 4 shows hard edge approximation of the measured solenoidal field.

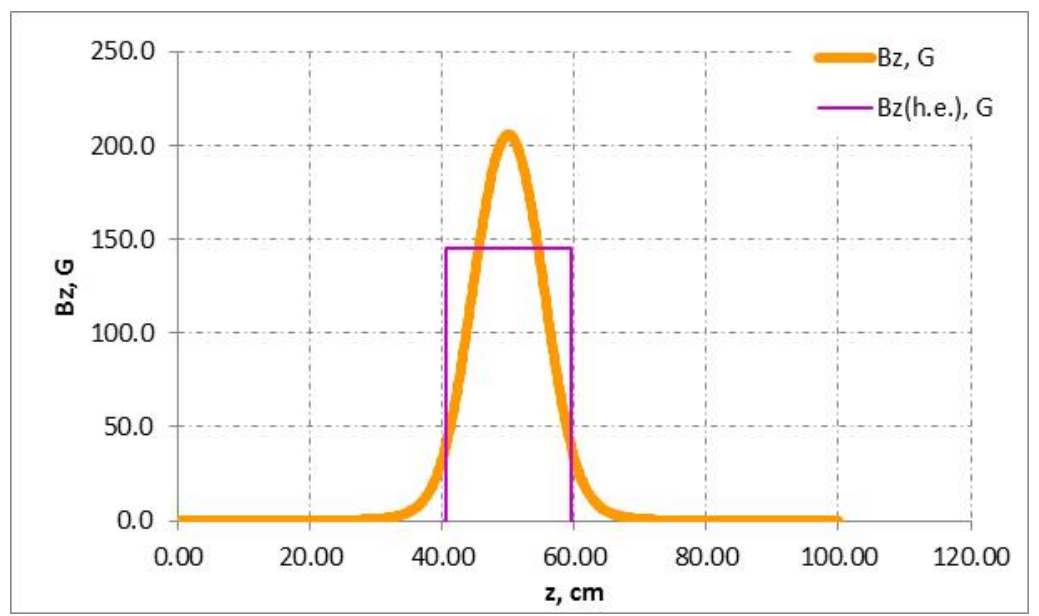

Figure 4: Measured (orange) and hard-edge approximation (pink) field of the CS solenoidal module.

To check the obtained approximation we compare results of simulations (3) to the beam trajectory found from a standard solenoid transfer matrix:

$$
M_{s o l}=\left(\begin{array}{cccc}
\frac{1+c}{2} & \frac{c}{k} & \frac{s}{2} & \frac{1-c}{k} \\
-\frac{k s}{4} & \frac{1+c}{2} & -k \frac{1-c}{4} & \frac{s}{2} \\
-\frac{s}{2} & -\frac{1-c}{k} & \frac{1+c}{2} & \frac{s}{k} \\
k \frac{1-c}{4} & -\frac{s}{2} & -\frac{k s}{4} & \frac{1+c}{2}
\end{array}\right), s \equiv \sin k L, \quad c \equiv \cos k L, \quad k=\frac{B}{B \rho}
$$

We find a perfect agreement between the beam trajectory in the hard-edge model and in the real distribution of the solenoid field (see Fig. 5).

\section{Effect of solenoid shift}

If the solenoid magnetic center is shifted from the ideal e-beam trajectory by $d_{x}$ and $d_{y}$, then beam trajectory at the exit of the solenoid is given by: 


$$
\left(\begin{array}{c}
x_{\text {shift }} \\
\theta x_{\text {shift }} \\
y_{\text {shift }} \\
\theta y_{\text {shift }}
\end{array}\right)=M_{\text {sol }}\left(\begin{array}{c}
d_{x} \\
0 \\
d_{y} \\
0
\end{array}\right)-\left(\begin{array}{c}
d_{x} \\
0 \\
d_{y} \\
0
\end{array}\right)
$$
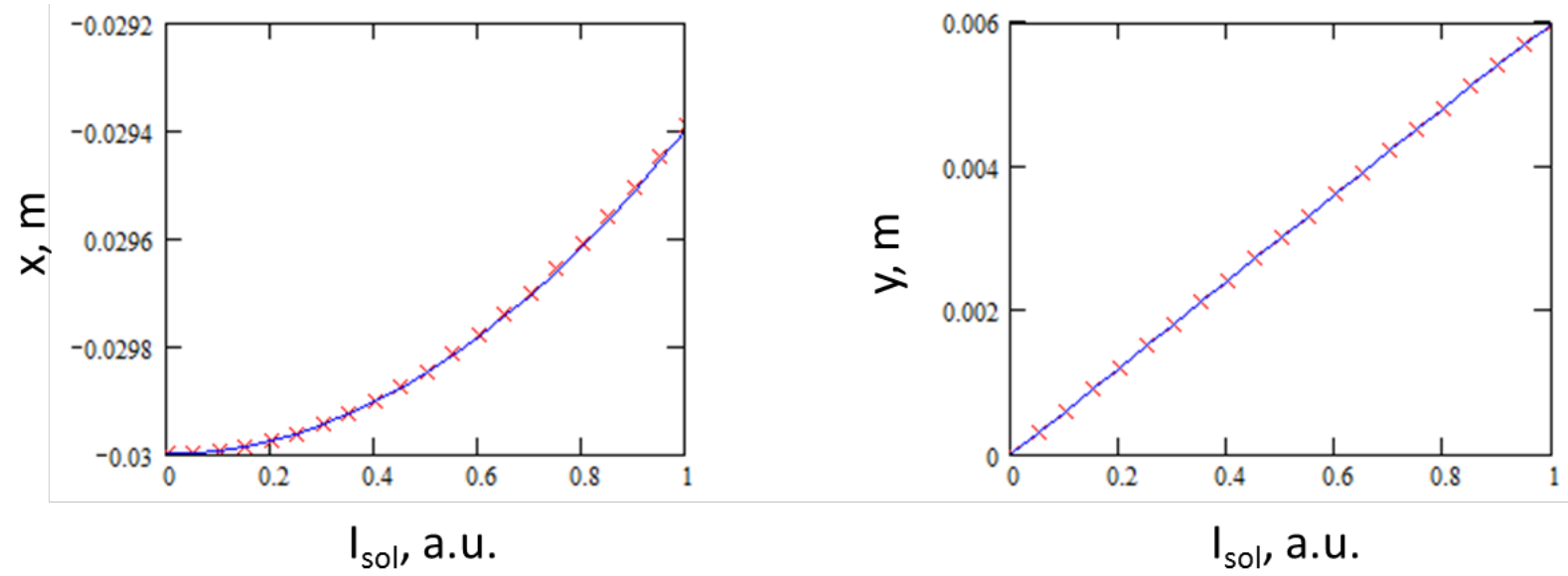

Figure 5: An example of the BPM readings simulated (red crosses) according to (3) and found in the hard-edge approximation (blue line). The BPM is located at $3 \mathrm{~m}$ from solenoid center. Beam trajectory at the entrance to the solenoid field region has an input angle $\theta_{x}=-10 \mathrm{mrad}$; the beam goes through $\mathrm{x}=0 \mathrm{~mm}$ at the center of the solenoid when the solenoid is switched off. $\mathrm{I}_{\text {sol }}=1$ corresponds to $205.7 \mathrm{G}$ peak field of the solenoid.

The readings of the BPM located at distance $z$ from the solenoid exit are given by:

$$
\left\{\begin{array}{l}
x_{\text {shiftBPM }}=x_{\text {shift }}+z \cdot \theta x_{\text {shift }} \\
y_{\text {shiftBPM }}=y_{\text {shift }}+z \cdot \theta y_{\text {shift }}
\end{array}\right.
$$

As Fig. 6 demonstrates results of Eq. (7) are in perfect agreement with simulations (3).
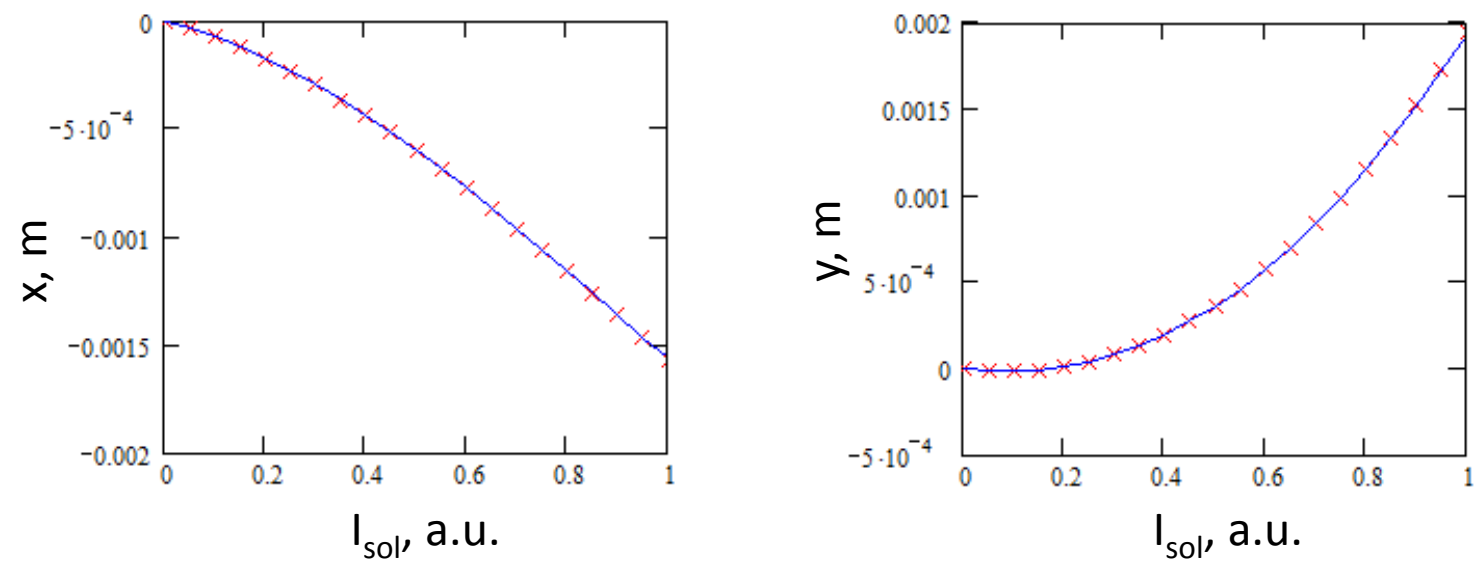
Figure 6: The BPM readings simulated (red crosses) according to (3) and found from (7) (blue line). The BPM is located at $3.2 \mathrm{~m}$ from the solenoid center. The solenoid is shifted by $d_{x}=2$ $\mathrm{mm}$ and $d_{y}=-3 \mathrm{~mm} . \mathrm{I}_{\text {sol }}=1$ corresponds to $205.7 \mathrm{G}$ peak field of the solenoid.

\section{Effect of solenoid inclination}

The simplest way to model the effect of an inclined solenoid on the e-beam trajectory is to switch into the inclined reference frame at solenoid entrance and switch back to the normal reference frame at solenoid exit. Then, in the hard-edge approximation the effect is given by:

$$
\left(\begin{array}{c}
x_{\text {incl }} \\
\theta x_{\text {incl }} \\
y_{\text {incl }} \\
\theta y_{\text {incl }}
\end{array}\right)=M_{\text {sol }}\left(\begin{array}{c}
-L \alpha_{x} / 2 \\
\alpha_{x} \\
-L \alpha_{y} / 2 \\
\alpha_{y}
\end{array}\right)-\left(\begin{array}{c}
L \alpha_{x} / 2 \\
\alpha_{x} \\
L \alpha_{y} / 2 \\
\alpha_{y}
\end{array}\right)
$$

The respective readings of the BPM located at distance $z$ from the solenoid exit are given by:

$$
\left\{\begin{array}{l}
x_{\text {inclBPM }}=x_{\text {incl }}+z \cdot \theta x_{\text {incl }} \\
y_{\text {inclBPM }}=y_{\text {incl }}+z \cdot \theta y_{\text {incl }}
\end{array}\right.
$$

Comparison of (9) to the simulations (3) show (see Fig. 7 for an example) the perfect agreement between analytic formulas (8)-(9) and the numerical simulation of beam motion through the real field of the CS solenoidal module.
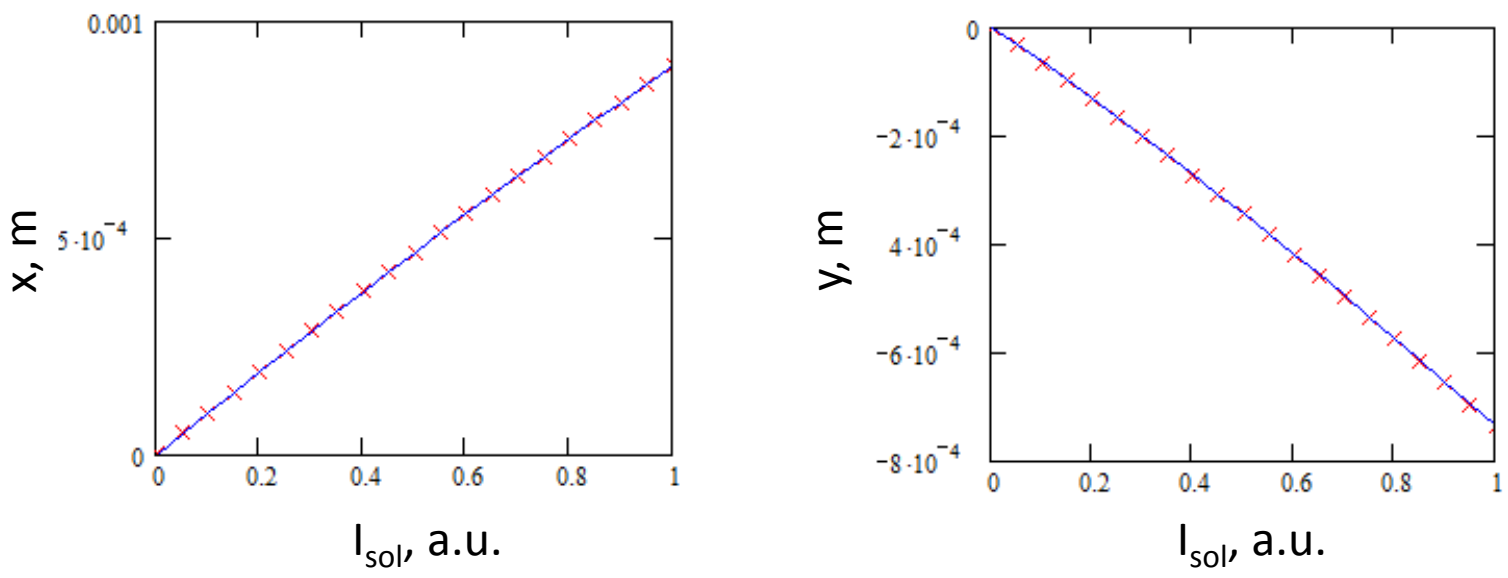

Figure 7: The BPM readings simulated (red crosses) according to (3) and found from (9) (blue line). The BPM is located at $3.2 \mathrm{~m}$ from the solenoid center. The solenoid is inclined by $\alpha_{x}=1 \mathrm{mrad}$ and $\alpha_{y}=1.5 \mathrm{mrad}$. $\mathrm{I}_{\text {sol }}=1$ corresponds to $205.7 \mathrm{G}$ peak field of the solenoid. 


\section{Effect of trajectory correctors}

Each cooling section solenoid is combined with transverse correctors. According to the procedure outlined in Section 1 these correctors will be set to compensate the effect of the residual earth field. In the model suggested in [1] these correctors will be providing $0.17 \mathrm{mrad}$ bending angle. Due to the $x-y$ rotation in solenoidal field there will be an additional effect of transverse correctors on beam trajectory for the turned on solenoid. While this effect is not too large (about 60 urad) we will take it into account nonetheless.

In these studies we assume that longitudinal distribution of the correctors' field repeats the field distribution of the solenoidal module.

Solving (1) in a hard-edge approximation and multiplying the obtained $x^{\prime} y^{\prime} y^{\prime}$ vector by a standard solenoid exit matrix we obtain the following formula for effect of transverse correctors in solenoidal field on the beam trajectory at solenoid exit:

$$
\left(\begin{array}{c}
x_{c o r r} \\
\theta x_{c o r r} \\
y_{c o r r} \\
\theta y_{c o r r}
\end{array}\right)=\left(\begin{array}{c}
\frac{B_{y}(c-1)+B_{x}(k L-s)}{B k} \\
\frac{B_{x}(1-c)-B_{y}(s+k L)}{2 B} \\
\frac{B_{x}(1-c)+B_{y}(k L-s)}{B k} \\
\frac{B_{y}(1-c)+B_{x}(s+k L)}{2 B}
\end{array}\right)-\left(\begin{array}{c}
-\frac{B_{y} L^{2}}{2 B \rho} \\
-\frac{B_{y} L}{B \rho} \\
\frac{B_{x} L^{2}}{2 B \rho} \\
\frac{B_{x} L}{B \rho}
\end{array}\right)
$$

The respective readings of the BPM located at distance $z$ from the solenoid exit are given by:

$$
\left\{\begin{array}{l}
x_{\text {corrBPM }}=x_{\text {corr }}+z \cdot \theta x_{\text {corr }} \\
y_{\text {corrBPM }}=y_{\text {corr }}+z \cdot \theta y_{\text {corr }}
\end{array}\right.
$$

Comparisons of (11) to the simulations (3) show (see Fig. 8) good agreement between analytic formulas and the numerical simulations.

It is also worth mentioning that in the model suggested in [1] the beam will be entering the CS solenoid with a small angle of about 20 urad. The effect from this angle on beam-based alignment procedure is negligibly small.

In Section 10 we will include these small angles into our numerical model but we will not account for the effect from these angles in the fitting functions.

\section{Additional considerations}

Let us expand the sum of the effects of solenoid shift and inclination: 


$$
\begin{aligned}
x_{\text {shiftBPM }}+x_{\text {inclBPM }}=\frac{B L}{B \rho} & {\left[\frac{d_{y}}{2}+\frac{\alpha_{y}}{2}\left(z+\frac{L}{2}\right)\right]-\left(\frac{B L}{B \rho}\right)^{2}\left[\frac{d_{x}}{4}\left(1+\frac{z}{L}\right)+\frac{\alpha_{x}}{8}\left(z+\frac{L}{3}\right)\right]+} \\
& +\left(\frac{B L}{B \rho}\right)^{3}\left[\frac{\alpha_{y}}{16} z-\frac{d_{y}}{4}\left(\frac{1}{3}+\frac{z}{2 L}\right)\right] \\
y_{\text {shiftBPM }}+y_{\text {inclBPM }}=- & \frac{B L}{B \rho}\left[\frac{d_{x}}{2}+\frac{\alpha_{x}}{2}\left(z+\frac{L}{2}\right)\right]-\left(\frac{B L}{B \rho}\right)^{2}\left[\frac{d_{y}}{4}\left(1+\frac{z}{L}\right)+\frac{\alpha_{y}}{8}\left(z+\frac{L}{3}\right)\right]+ \\
+ & \left(\frac{B L}{B \rho}\right)^{3}\left[\frac{d_{x}}{4}\left(\frac{1}{3}+\frac{z}{2 L}\right)-\frac{\alpha_{x}}{16} z\right]
\end{aligned}
$$
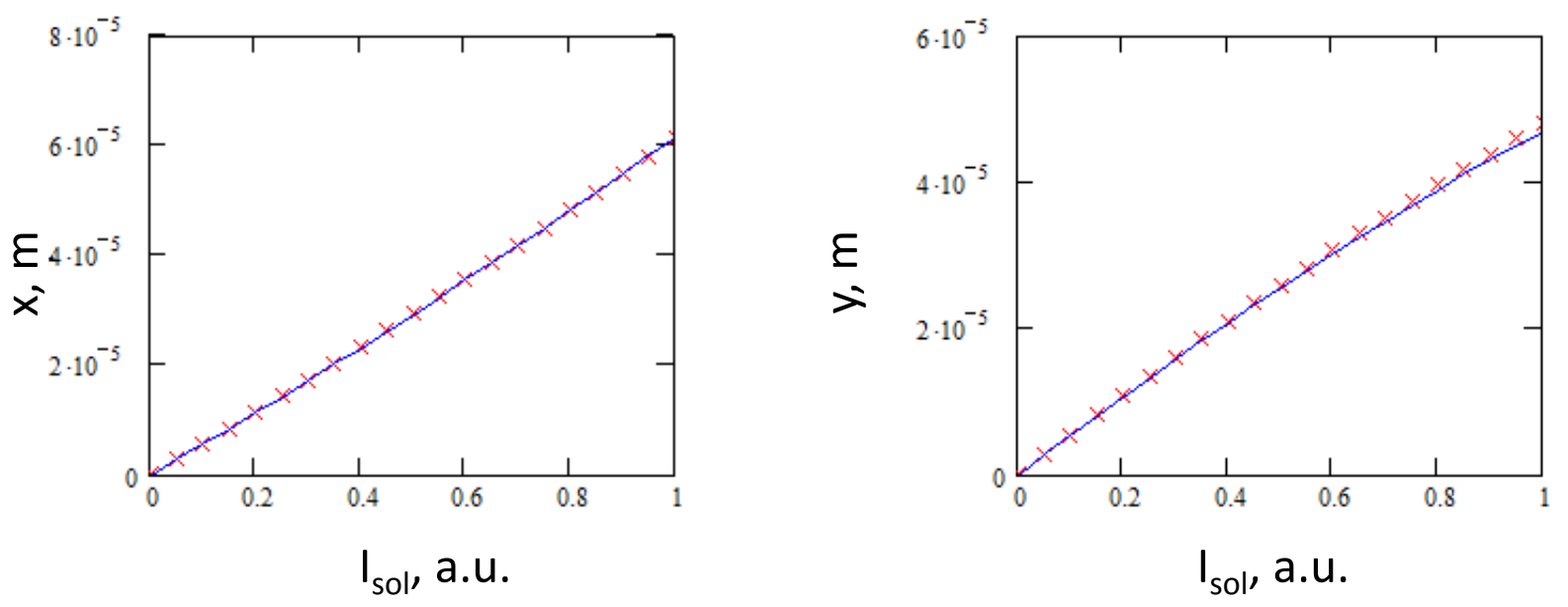

Figure 8: The BPM readings simulated (red crosses) according to (3) and found from (11) (blue line). The BPM is located at $3.2 \mathrm{~m}$ from the solenoid center. Both transverse correctors inside the solenoid are set to provide $0.17 \mathrm{mrad}$ angle. $\mathrm{I}_{\text {sol }}=1$ corresponds to $205.7 \mathrm{G}$ peak field of the solenoid.

Equation (12) shows that the effect of the shift of a solenoid just upstream of the BPM on this BPM readings can be indistinguishable from the effect of the inclination of the solenoid located at some distance upstream of the BPM.

Indeed, Fig. 9 demonstrates that if all CS solenoids are turned on together then from BPM readings it would be hard to determine whether the far-away solenoid is inclined or the nearby one is shifted.

Therefore, it is critical for proper alignment of the e-beam trajectory through the cooling section to be able to have an individual control of each CS solenoid. 

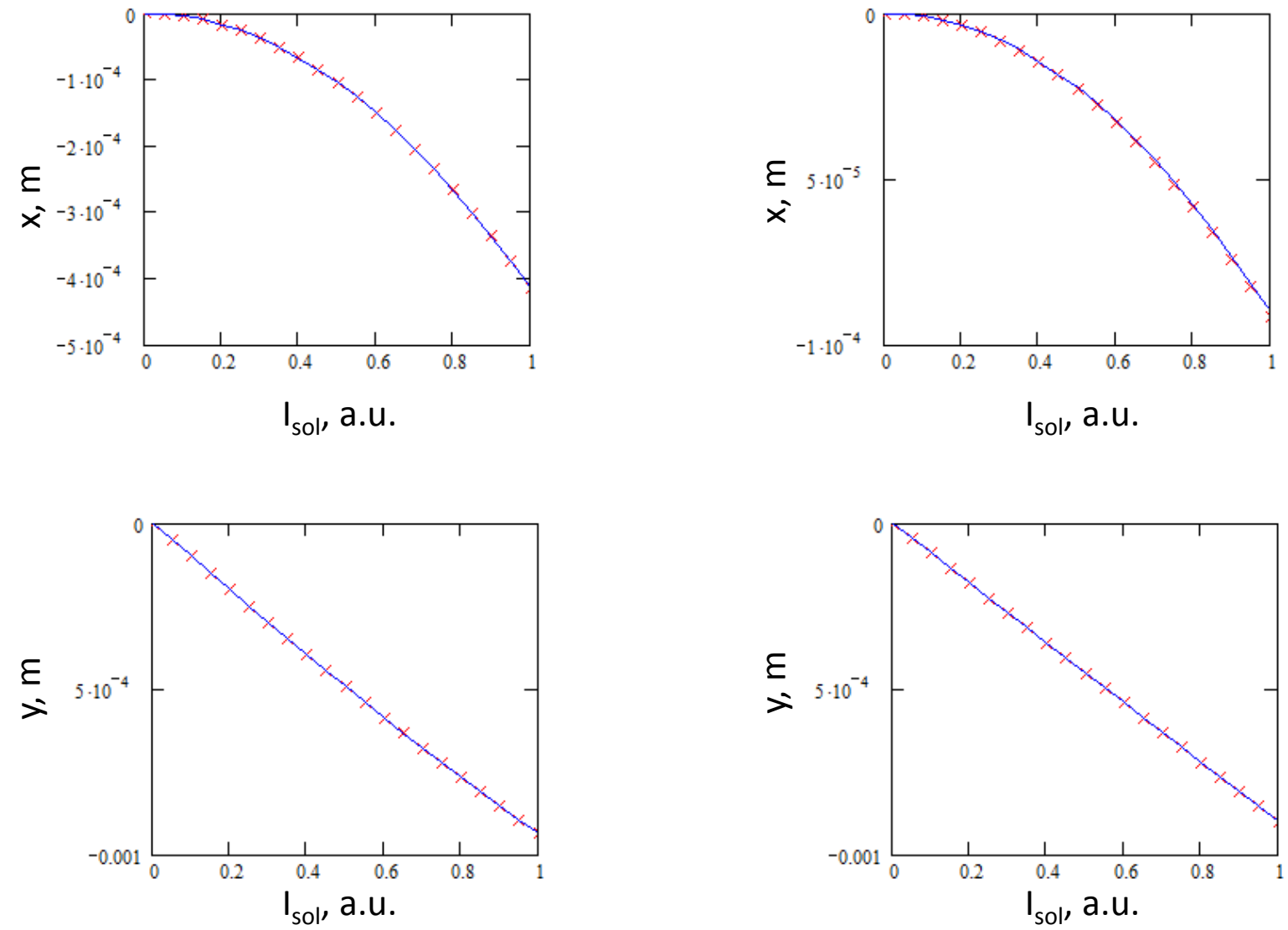

Figure 9: Effects of the solenoid ( $20 \mathrm{~cm}$ upstream of the BPM) shifted by $5 \mathrm{~mm}$ (left plots) and of the solenoid (3.2 m upstream of the BPM) inclined by $1.5 \mathrm{mrad}$ (right plots).

\section{Solenoid beam-based alignment}

To find the inclination and the shift of each CS solenoid we will measure dependence of the readings of the downstream BPM on the solenoid current and fit obtained dependence with the following fitting functions:

$$
\left\{\begin{array}{l}
x_{B P M}\left(d_{x}, d_{y}, \alpha_{x}, \alpha_{y}, B\right)=x_{\text {shiftBPM }}\left(d_{x}, d_{y}, B\right)+x_{\text {inclBPM }}\left(\alpha_{x}, \alpha_{y}, B\right)+x_{\text {corr } B P M}(B) \\
y_{B P M}\left(d_{x}, d_{y}, \alpha_{x}, \alpha_{y}, B\right)=y_{\text {shiftBPM }}\left(d_{x}, d_{y}, B\right)+y_{\text {inclBPM }}\left(\alpha_{x}, \alpha_{y}, B\right)+y_{\text {corrBPM }}(B)
\end{array}\right.
$$

The fit will be obtained by minimizing:

$$
\chi^{2}=\frac{1}{N-4} \sum_{n=1}^{N}\left\{\left[\frac{x_{n}-x_{B P M}\left(d_{x}, d_{y}, \alpha_{x}, \alpha_{y}, B_{n}\right)}{\sigma_{x}}\right]^{2}+\left[\frac{y_{n}-y_{B P M}\left(d_{x}, d_{y}, \alpha_{x}, \alpha_{y}, B_{n}\right)}{\sigma_{y}}\right]^{2}\right\}
$$

Here $x_{n}$ and $y_{n}$ are BPM readings corresponding to $B_{n}$, and $\sigma_{x}$ and $\sigma_{y}$ are RMS spread of BPM readings. 
We modeled the outlined beam-based alignment process. We used (3) to simulate $x_{n}$ and $y_{n}$. We assumed $10 \mathrm{um}$ for both $\sigma_{x}$ and $\sigma_{y}$. We chose $\mathrm{N}=21$.

As a result, even if we use just one BPM (at $3.2 \mathrm{~m}$ from solenoid center in our model) for the fitting, the solenoid shift can be found with $~ 90$ um accuracy and solenoid inclination can be found with an accuracy of about 70 urad.

We can improve this result by using two BPMs for fitting - one at $3.2 \mathrm{~m}$ downstream of solenoid center and one at $6.2 \mathrm{~m}$ downstream of the solenoid. In that case we obtain the solenoid shift with 40 um accuracy and solenoid inclination with 30 urad accuracy. Figure 10 shows an example of the fitting performed with two BPMs.
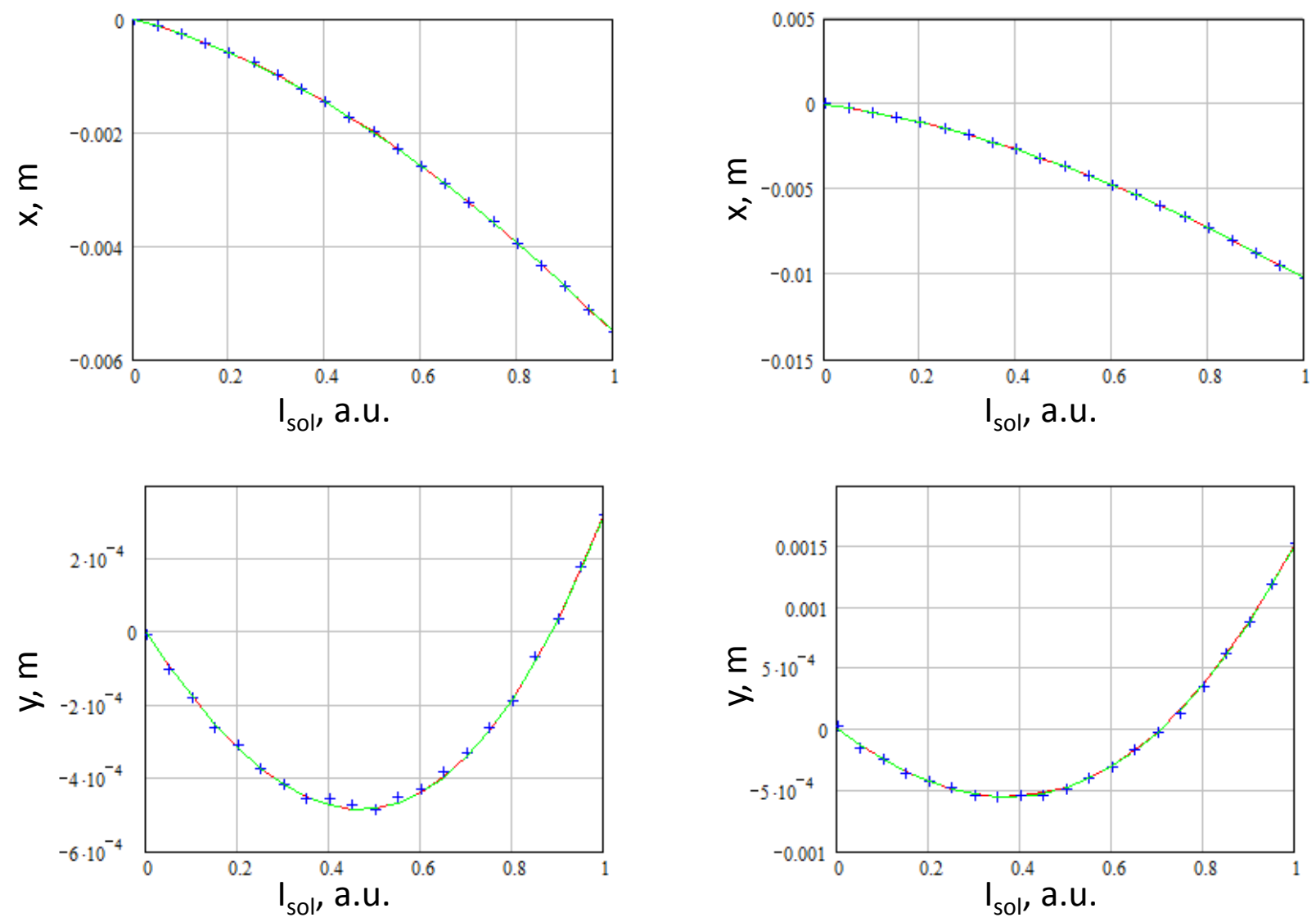

Figure 10: Fitting (green curve) BPM readings (blue crosses) with two BPMs. First BPM (left plots) is located at $3.2 \mathrm{~m}$ downstream of the solenoid center and second BPM (right plot) is located at $6.2 \mathrm{~m}$ downstream of the solenoid. In this example $d_{x}=5 \mathrm{~mm}, d_{y}=-2 \mathrm{~mm}, \alpha_{x}=$ $1.5 \mathrm{mrad}, \alpha_{y}=-3 \mathrm{mrad}$. $\mathrm{I}_{\text {sol }}=1$ corresponds to $205.7 \mathrm{G}$ peak field of the solenoid.

\section{Conclusion}

We considered the steps required to align the electron beam trajectory through the LEReC cooling section. 
We devised a detailed procedure for the beam-based alignment of the cooling section solenoids.

We showed that it is critical to have an individual control of each CS solenoid current.

Finally, we modeled the alignment procedure and showed that with two BPM fitting the solenoid shift can be measured with 40 um accuracy and the solenoid inclination can be measured with 30 urad accuracy. These accuracies are well within the tolerances of the cooling section solenoid alignment.

\section{References}

[1] S. Seletskiy et al., BNL-112084-2016-IR, 2016.

[2] G. Mahler et al., 010606318 SOW-01, 2014.

[3] W. Meng, compensating_sole_modified_2.xlsx.

[4] A. Jain, Results of Magnetic Measurements in LEReC Solenoids, 2015.

[5] S. Seletskiy, Attainment of Electron Beam Suitable for Medium Energy Electron Cooling, PhD thesis, 2005. 